Research Article

\title{
On the Existence and Uniqueness Results for Fuzzy Linear and Semilinear Fractional Evolution Equations Involving Caputo Fractional Derivative
}

\author{
Ali El Mfadel $(\mathbb{D}$, Said Melliani $\mathbb{D}$, and M'hamed Elomari \\ Laboratory of Applied Mathematics and Scientific Computing, Sultan Moulay Slimane University, Beni Mellal, Morocco \\ Correspondence should be addressed to Ali El Mfadel; elmfadelali@gmail.com
}

Received 6 July 2021; Revised 4 August 2021; Accepted 9 August 2021; Published 7 September 2021

Academic Editor: Serena Matucci

Copyright (C) 2021 Ali El Mfadel et al. This is an open access article distributed under the Creative Commons Attribution License, which permits unrestricted use, distribution, and reproduction in any medium, provided the original work is properly cited.

In this manuscript, we establish new existence and uniqueness results for fuzzy linear and semilinear fractional evolution equations involving Caputo fractional derivative. The existence theorems are proved by using fuzzy fractional calculus, Picard's iteration method, and Banach contraction principle. As application, we conclude this paper by giving an illustrative example to demonstrate the applicability of the obtained results.

\section{Introduction}

Fuzzy fractional calculus and fuzzy fractional differential equations are a natural way to model dynamical systems subject to uncertainties. In the past few years, many works have been done by several authors in the theory of fuzzy fractional differential equations (see [1-3]). This theory has been proposed to handle uncertainty due to incomplete information that appears in many mathematical or computer models of some deterministic real-world phenomena. Recently, fractional differential equations have attracted a considerable interest both in mathematics and in applications such as material theory, transport processes, fluid flow phenomena, earthquakes, solute transport, chemistry, wave propagation, signal theory, biology, electromagnetic theory, thermodynamics, mechanics, geology, astrophysics, economics heat conduction in materials with memory, and control theory (see basic books and interesting papers in [4-8]).

In many cases, when a real physical phenomenon is modelled by a fractional initial value problem, we cannot usually be sure that the model is perfect. For example, the initial value of this problem may not be known precisely. In order to get a perfect model with a precise initial condition, Agarwal et al. in [9] proposed the concept of solutions for fuzzy fractional differential equations. Arshad and Lupulescu in [10] proved some results on the existence and uniqueness of solution for the fuzzy fractional differential equations under Hukuhara fractional Riemann-Liouville differentiability. Later, Alikhani and Bahrami in [11] have proved the existence and uniqueness results for nonlinear fuzzy fractional integral and integrodifferential equations by using the method of upper and lower solutions. The authors in $[12,13]$ discussed the concepts about generalized Hukuhara fractional RiemannLiouville and Caputo differentiability of fuzzy-valued functions, and the equivalence between fuzzy fractional differential equation and fuzzy fractional integral equation is discussed in [14]. For many basic works related to the theory of fractional differential equations and fuzzy fractional differential equations, we refer the readers to the articles [15-20] and references therein.

Motivated by the above works, in the present paper, we study the existence result of solution for the following fuzzy linear fractional evolution equation:

$$
\left\{\begin{array}{l}
{ }^{c} D_{t_{0}^{+}}^{q} x(t)=A(t) x(t), \quad t \in J=\left[t_{0}, T\right], \\
x\left(t_{0}\right)=x_{0},
\end{array}\right.
$$

and for the following fuzzy semilinear fractional evolution equation: 


$$
\left\{\begin{array}{l}
{ }^{c} D_{t_{0}^{+}}^{q} x(t)=A(t) x(t)+f(t, x(t)), \quad t \in J=\left[t_{0}, T\right], \\
x\left(t_{0}\right)=x_{0},
\end{array}\right.
$$

where ${ }^{c} D_{t^{+}}^{q}$ is the fuzzy Caputo derivative of $x(t)$ at order $0<q<1, t_{0} \geq 0, T>0, A(t)$ is a bounded linear operator, and $f$ is a fuzzy continuous function.

The paper is organized as follows. In Section 2, we give some basic properties of fuzzy sets, operations of fuzzy numbers, and some detailed definitions of fuzzy fractional integral and fuzzy fractional derivative which will be used in the rest of this paper. In Section 3, we introduce the existence and uniqueness results of solution for the fuzzy linear fractional evolution equation (1). In Section 4, we discussed the existence and uniqueness results for the fuzzy semilinear fractional evolution equation (2). An illustrative example is presented in Section 5 followed by conclusion and future work in Section 6.

\section{Preliminaries}

In this section, we will briefly give some of notations, definitions, and results from the literature of fuzzy set theory and fuzzy fractional calculus which will be used in the rest of this paper.

Definition 1 (see [21]). A fuzzy number is mapping $u: \mathbf{R}$ $\longrightarrow[0,1]$ such that

(1) $u$ is upper semicontinuous

(2) $u$ is normal; that is, there exist $x_{0} \in \mathbf{R}$ such that $u$ $\left(x_{0}\right)=1$

(3) $u$ is fuzzy convex, that is, $u(\lambda x+(1-\lambda) y) \geq \min$ $\{u(x), u(y)\}$ for all $x, y \in \mathbf{R}$ and $\lambda \in[0,1]$

(4) $\{x \in \mathbf{R}, \bar{u}(x)>0\}$ is compact

The $\alpha$-cut of a fuzzy number $u$ is defined as follows:

$$
[u]^{\alpha}=\{x \in \mathbf{R} \mid u(x) \geq \alpha\}
$$

Moreover, we also can present the $\alpha$-cut of fuzzy number $u$ by $[u]^{\alpha}=\left[u_{l}(\alpha), u_{r}(\alpha)\right]$.

Example 1. Let $u$ be a fuzzy number defined by the following function:

$$
\mu_{u}(x)= \begin{cases}x-1, & x \in[1,2] \\ -x+3, & x \in[2,3] \\ 0, & \text { elswhere. }\end{cases}
$$

If $\alpha=1$, then the $\alpha$-cut of the fuzzy number $u$ is given by $[u]^{\alpha}=[u]^{1}=\{2\}$.
Notations.

(i) We denote by $E^{1}$ the collection of all fuzzy numbers

(ii) We denote by $C\left(J, E^{1}\right)$ the space of all fuzzy-valued functions which are continuous on $J$

(iii) We denote by $\mathbf{P}_{c}(\mathbf{R})$ the set of all bounded and closed intervals of $\mathbf{R}$

(iv) We denote also by $0_{E^{1}}$ the fuzzy zero defined by

$$
0_{E^{1}}(x)= \begin{cases}1, & \text { if } x=0 \\ 0, & \text { elswhere }\end{cases}
$$

Definition 2 (see [14]). Let $\alpha \in[0,1]$ and $u \in E^{1}$ such that $[u]^{\alpha}=\left[u_{l}(\alpha), u_{r}(\alpha)\right]$.

We define the diameter of $\alpha$-level set $[u]^{\alpha}$ of the fuzzy set $u$ as follows:

$$
d\left([u]^{\alpha}\right)=u_{r}(\alpha)-u_{l}(\alpha)
$$

Definition 3 (see [14]). The generalized Hukuhara difference of two fuzzy numbers $u, v \in E^{1}$ is defined as follows:

$$
u \ominus_{g H} v=w \Longleftrightarrow \begin{cases}\text { (i) } & u=v+w, \\ \text { or } & \\ \text { (ii) } & v=u+(-w) .\end{cases}
$$

Property 4 (see [22]). If $u \in E^{1}$ and $v \in E^{1}$, then the following properties hold:

(1) If $u \ominus_{g H} v$ exists then it is unique

(2) $u \ominus_{g H} u=0_{E^{1}}$

(3) $(u+v) \ominus_{g H} v=u$

(4) $u \ominus_{g H} v=0_{E^{1}} \Leftrightarrow u=v$

Definition 5 (see [21]). According to Zadeh's extension principle, the addition on $E^{1}$ is defined by

$$
(u \oplus v)(z)=\sup _{z=x+y} \min \{u(x), v(y)\}
$$

And scalar multiplication of a fuzzy number is given by

$$
(k e u)(x)= \begin{cases}u(x / k), & k \in \mathbf{R}-\{0\} \\ 0_{E^{1}}, & k=0 .\end{cases}
$$

Remark 6 (see [23]). Let $u, v \in E^{1}$ and $\alpha \in[0,1]$; then, we have 


$$
\begin{gathered}
{[u+v]^{\alpha}=[u]^{\alpha}+[v]^{\alpha},} \\
{[u-v]^{\alpha}=\left[u_{1}^{\alpha}-v_{2}^{\alpha}, u_{2}^{\alpha}-v_{1}^{\alpha}\right],} \\
{[k u]^{\alpha}=k[u]^{\alpha}= \begin{cases}{\left[k u_{1}^{\alpha}, k u_{2}^{\alpha}\right],} & \text { if } k \geq 0, \\
{\left[k u_{2}^{\alpha}, k u_{1}^{\alpha}\right],} & \text { if } k \leq 0,\end{cases} } \\
{[u v]^{\alpha}=\left[\min u_{1}^{\alpha} v_{1}^{\alpha}, u_{1}^{\alpha} v_{2}^{\alpha}, u_{2}^{\alpha} v_{1}^{\alpha}, u_{2}^{\alpha} v_{2}^{\alpha}, \max u_{1}^{\alpha} v_{1}^{\alpha}, u_{1}^{\alpha} v_{2}^{\alpha}, u_{2}^{\alpha} v_{1}^{\alpha}, u_{2}^{\alpha} v_{2}^{\alpha}\right] .}
\end{gathered}
$$

Definition 7 (see [24]). Let $u, v \in E^{1}$ and $\alpha \in[0,1]$; then, the Hausdorf distance between $u$ and $v$ is given by

$$
D(u, v)=\sup _{\alpha \in[0,1]} \max \left\{\left|u_{l}(\alpha)-v_{l}(\alpha)\right|,\left|u_{r}(\alpha)-v_{r}(\alpha)\right|\right\} .
$$

Proposition 8 (see [25]). D is a metric on $E^{1}$ and has the following properties:

(1) $\left(E^{1} ; D\right)$ is a complete metric space

(2) $D(u+w, v+w)=D(u, v), \forall u, v, w \in E^{1}$

(3) $D(k u, k v)=|k| D(u, v), \forall u, v \in E^{l}$ and $k \in R$

(4) $D(u+w, v+z) \leq D(u, v)+D(w, z), \forall u, v, w, z \in E^{1}$

Remark 9. Let $x, y \in C\left(\left[J, E^{1}\right)\right.$. It is easy to see that the space $\left(C\left(J, E^{1}\right), D_{s}\right)$ is a Banach space where

$$
D_{s}(x, y)=\sup _{s \in J} D(x(s), y(s))
$$

Definition 10 (see [26]). Let $f: J \longrightarrow E^{1}$ and $t_{0} \in J$. We say that $f$ is Hukuhara differentiable at $t_{0}$ if there exists $f^{\prime}\left(t_{0}\right)$ $\in E^{1}$ such that

$$
f^{\prime}\left(t_{0}\right)=\lim _{h \longrightarrow 0^{+}} \frac{f\left(t_{0}+h\right) \ominus_{g H} f\left(t_{0}\right)}{h}=\lim _{h \longrightarrow 0^{-}} \frac{f\left(t_{0}\right) \ominus_{g H} f\left(t_{0}-h\right)}{h} .
$$

Definition 11 (see [25]).

(1) A function $F: J \longrightarrow E^{1}$ is strongly measurable if $\forall$ $\alpha \in[0,1]$, the set-valued mapping $F_{\alpha}: J \longrightarrow \mathscr{P}_{c}(\mathbf{R})$ defined by $F_{\alpha}(t)=[F(t)]^{\alpha}$ is Lebesgue measurable

(2) A function $F: J \longrightarrow E^{1}$ is called integrably bounded, if there exists an integrable function $h$ such that $|x|<h(t) \forall x \in F_{0}(t)$

Definition 12. Let $F: J \longrightarrow E^{1}$. The integral of $F$ on $J$ denoted by $\int_{J} F(t) d t$ is defined by

$$
\begin{aligned}
{\left[\int_{J} F(t) d t\right]^{\alpha} } & =\int_{J} F_{\alpha}(t) d t \\
& =\left\{\int_{J} f(t) d t \mid f: J \longrightarrow \mathbf{R} \text { is a measurable selection for } F_{\alpha}\right\}
\end{aligned}
$$

for all $\alpha \in[0,1]$.
Proposition 13 (see [25]). Let $F: J \longrightarrow E^{1}$ be a fuzzy function. If $F$ is strongly measurable and integrably bounded, then it is integrable.

2.1. Fractional Integral and Fractional Derivative of Fuzzy Function

Proposition 14. If $u \in E^{1}$, then the following properties hold:

(1) $[u]^{\beta} \subset[u]^{\alpha}$ if $0 \leq \alpha \leq \beta \leq 1$.

(2) If $\alpha_{n} \subset[0,1]$ is a nondecreasing sequence which converges to $\alpha$, then

$$
[u]^{\alpha}=\bigcap_{n \geq 1}[u]^{\alpha_{n}} .
$$

Conversely, if $A^{\alpha}=\left\{\left[u_{1}^{\alpha}, u_{2}^{\alpha}\right] ; \alpha \in[0,1]\right\}$ is a family of closed real intervals verifying (1) and (2), then $A^{\alpha}$ defined a fuzzy number $u \in E^{1}$ such that $[u]^{\alpha}=A^{\alpha}$.

Let $0<q<1$; the fractional integral of order $q$ of a real function $g: J \longrightarrow R$ is given by

$$
I_{t_{0}^{+}}^{q} g(t)=\frac{1}{\Gamma(q)} \int_{0}^{t}(t-s)^{q-1} g(s) d s
$$

Let $f(t) \in L\left(J, E^{1}\right) \quad$ such that $f(t)=\left[f_{1}^{\alpha}(t), f_{2}^{\alpha}(t)\right]$. Suppose that $f_{1}^{\alpha}, f_{2}^{\alpha} \in L(J, R)$ for all $\alpha \in[0,1]$ and let

$$
A^{\alpha}=\left[\frac{1}{\Gamma(q)} \int_{0}^{t}(t-s)^{q-1} f_{1}^{\alpha}(s) d s, \frac{1}{\Gamma(q)} \int_{0}^{t}(t-s)^{q-1} f_{2}^{\alpha}(s) d s\right],
$$

where $\Gamma($.$) is the Euler gamma function.$

We have the following lemma.

Lemma 15 (see [10]). The family $\left\{A^{\alpha} ; \alpha \in[0,1]\right\}$ given by (17) defined a fuzzy number $u \in E^{1}$ such that $[u]^{\alpha}=A^{\alpha}$.

Definition 16 (see [10]). Let $f(t) \in L\left(J, E^{1}\right)$.

The fuzzy fractional integral at order $q \in[0,1]$ of $f$ denoted by

$$
I_{t_{0}^{+}}^{q} f(t)=\frac{1}{\Gamma(q)} \int_{t_{0}}^{t}(t-s)^{q-1} f(s) d s
$$

is defined by

$$
\left[I_{t_{0}^{+}}^{q} f(t)\right]^{\alpha}=\left[I_{t_{0}^{+}}^{q} f_{l}(t ; \alpha), I_{t_{0}^{+}}^{q} f_{r}(t ; \alpha)\right]
$$

Proposition 17 (see [10]). Let $f, g \in L\left(J, E^{l}\right)$ and $b \in E^{l}$; then, we have

(1) $I_{t_{0}^{+}}^{q}(b f)(t)=b I_{t_{0}^{+}}^{q} f(t)$

(2) $I_{t_{0}^{+}}^{q}(f+g)(t)=I_{t_{0}^{+}}^{q} f(t)+I_{t_{0}^{+}}^{q} g(t)$ 
(3) $I^{q_{1}} I^{q_{2}} f(t)=I^{q_{1}+q_{2}} f(t)$, where $\left(q_{1}, q_{2}\right) \in[0,1]^{2}$

Example 2. Let $x: J \longrightarrow E^{1}$ be a constant fuzzy function such that $x(t)=u \in E^{1}$.

If $[u]^{\alpha}=\left[u_{\alpha}^{1}, u_{\alpha}^{2}\right]$, then

$$
\begin{aligned}
& {\left[I_{t_{0}^{+}}^{q} x(t)\right]^{\alpha}=\left[\frac{1}{\Gamma(q)} \int_{t_{0}}^{t}(t-s)^{q-1} u_{\alpha}^{1}(s) d s, \frac{1}{\Gamma(q)} \int_{t_{0}}^{t}(t-s)^{q-1} u_{\alpha}^{2}(s) d s\right],} \\
& {\left[I_{t_{0}^{+}}^{q} x(t)\right]^{\alpha}=\frac{t^{q}}{\Gamma(q+1)}\left[u_{\alpha}^{1}, u_{\alpha}^{2}\right],} \\
& {\left[I_{t_{0}^{+}}^{q} x(t)\right]^{\alpha}=\frac{t^{q}}{\Gamma(q+1)}[u]^{\alpha} .}
\end{aligned}
$$

Definition 18 (see [13]). Let $f \in C\left(J, E^{1}\right) \cap L\left(J, E^{1}\right)$. The function $f$ is called fuzzy Caputo fractional differentiable of order $0<q<1$ at $t$ if there exists an element ${ }^{c} D_{t_{0}^{+}}^{q} f(t) \in E^{1}$ such that

$$
{ }^{c} D_{t_{0}^{+}}^{q} f(t)=\frac{1}{\Gamma(q)} \int_{t_{0}}^{t}(t-s)^{q-1} f^{\prime}(s) d s .
$$

Remark 19 [13]. Since $[f(t)]^{\alpha}=\left[f_{l}(t ; \alpha), f_{r}(t ; \alpha)\right]$ for each $\alpha \in[0,1]$, then

$$
\left[{ }^{c} D_{t_{0}^{+}}^{q} f(t)\right]^{\alpha}=\left[{ }^{c} D_{t_{0}^{+}}^{q} f_{l}(t ; \alpha),{ }^{c} D_{t_{0}^{+}}^{q} f_{r}(t ; \alpha)\right],
$$

where

$$
\begin{gathered}
{ }^{c} D_{t_{0}^{+}}^{q} f_{l}(t ; \alpha):=\frac{1}{\Gamma(\alpha)} \int_{0}^{t}(t-s)^{\alpha-1} f_{l^{\prime}}(s, \alpha) d s, \\
{ }^{c} D_{t_{0}^{+}}^{q} f_{r}(t ; \alpha):=\frac{1}{\Gamma(\alpha)} \int_{t_{0}}^{t}(t-s)^{\alpha-1} f_{r}^{\prime}(s, \alpha) d s .
\end{gathered}
$$

Example 3. Let $x(t)=u \in E^{1}$. If $[u]^{\alpha}=\left[u_{\alpha}^{1}, u_{\alpha}^{2}\right]$, then

$$
\begin{aligned}
{\left[{ }^{c} D_{t_{0}^{+}}^{q} x(t)\right]^{\alpha} } & =\left[\frac{1}{\Gamma(q)} \int_{t_{0}}^{t}(t-s)^{q-1}\left(u_{\alpha}^{1}\right)^{\prime} d s, \frac{1}{\Gamma(q)} \int_{t_{0}}^{t}(t-s)^{q-1}\left(u_{\alpha}^{2}\right)^{\prime} d s\right], \\
{\left[{ }^{c} D_{t_{0}^{+}}^{q} x(t)\right]^{\alpha} } & =\{0\}, \\
{ }^{c} D_{t_{0}^{+}}^{q} x(t) & =0_{E^{1}} .
\end{aligned}
$$

\section{Fuzzy Linear Fractional Evolution Equation}

Definition 20 (see [14]). A fuzzy function $x: J \longrightarrow E^{1}$ is called $d$-increasing $(d$-decreasing) on $J$ if for every $\alpha \in[0,1]$, the real function $t \longrightarrow d\left([x(t)]^{\alpha}\right)$ is nondecreasing (nonincreasing), respectively.

Remark 21. If $x: J \longrightarrow E^{1}$ is $d$-increasing or $d$-decreasing on $J$, then we say that $x(t)$ is $d$-monotone on $J$.
Definition 22. A fuzzy function $x(t)$ is a solution of problem (1) if and only if

(1) $x(t)$ is continuous and $x(t) \in D(A(t))$ for all $t \in J$

(2) ${ }^{c} D_{t_{0}^{+}}^{q} x(t)$ exists and continuous on $J$, where $0<q<1$

(3) $x(t)$ satisfies (1)

Lemma 23. A $d$-monotone fuzzy function $x(t)$ is a solution of problem (1) if and only if

(1) $x(t)$ is continuous and $x(t) \in D(A(t))$ for all $t \in J$

(2) $x(t)$ satisfies the following integral equation

$$
\left.x(t) \ominus_{g H} x_{0}=\frac{1}{\Gamma(q)} \int_{t_{0}}^{t}(t-s)^{q-1} A(s) x(s)\right) d s
$$

(3) The function $t \longrightarrow I_{t_{0}^{+}}^{q} A(t) x(t)$ is d-increasing on $J$

Proof. See the proof of Theorem 3 in [14].

Theorem 24. Assume that

$H_{1} A(t)$ is a bounded linear operator on $E^{1}$ for each $t \in J$. $\mathrm{H}_{2}$ The function $t \longrightarrow A(t)$ is continuous.

If the assumptions $\mathrm{H}_{1}-\mathrm{H}_{2}$ hold, then problem (1) has a unique solution on $\mathrm{J}$.

Proof. To show that problem (1) has a unique solution defined on $J$, we use Picard's iteration method (see [27]).

Let

$$
S=\sup _{t \in J} D\left(A(t), 0_{E^{1}}\right),
$$

and let $T: C\left(J, E^{1}\right) \longrightarrow C\left(J, E^{1}\right)$ be the operator defined as follows:

$$
T x(t) \ominus_{g H} x_{0}=\frac{1}{\Gamma(\alpha)} \int_{t_{0}}^{t}(t-s)^{q-1} A(s) x(s) d s .
$$

Let $x, y \in C\left(J, E^{1}\right)$ : then, we have

$$
D_{s}(T x(t), T y(t)) \leq \frac{S\left(T-t_{0}\right)^{q}}{\Gamma(q+1)} D_{s}(T x, T y),
$$

and by induction, we can write

$$
D\left(T^{n} x(t), T^{n} y(t)\right) \leq \frac{\left(\left(S\left(T-t_{0}\right)^{q}\right) /(\Gamma(q+1))\right)^{n}}{n !} D_{s}\left(T^{n} x, T^{n} y\right) .
$$


It follows that

$$
D_{s}\left(T^{n} x, T^{n} y\right) \leq \frac{\left(\left(S\left(T-t_{0}\right)^{q}\right) /(\Gamma(q+1))\right)^{n}}{n !} D_{s}\left(T^{n} x, T^{n} y\right) .
$$

Finally, since $\left(\left(\left(S\left(T-t_{0}\right)^{q}\right) /(\Gamma(q+1))\right)^{n} / n !\right)<1$ for large $n$, then by the well-known generalization of the Banach contraction principle, the operator $T$ has a unique fixed point $x \in C\left(J, E^{1}\right)$ which is the solution of problem (1).

\section{Fuzzy Semilinear Fractional Evolution Equation}

Definition 25. A fuzzy function $x(t)$ is a solution of problem (2) if and only if

(1) $x(t)$ is continuous and $x(t) \in D(A(t))$ for all $t \in J$

(2) $x(t)$ satisfies (2)

Lemma 26. A d-monotone fuzzy function $x(t)$ is a solution of problem (2) if and only if

(1) $x(t)$ is continuous and $x(t) \in D(A(t))$ for all $t \in J$

(2) $x(t)$ satisfies the following integral equation:

$$
\begin{aligned}
x(t) \ominus_{g H} x_{0}= & \left.\frac{1}{\Gamma(q)} \int_{t_{0}}^{t}(t-s)^{q-1} A(s) x(s)\right) d s \\
& +\frac{1}{\Gamma(q)} \int_{t_{0}}^{t}(t-s)^{q-1} f(s, x(s)) d s
\end{aligned}
$$

(3) The function $t \longrightarrow I_{t_{0}^{+}}^{q}(A(t) x(t)+f(t, x(t))$ is $d$ -increasing on $J$

Proof. See the proof of Theorem 3 in [14].

Theorem 27. Assume that

$\mathrm{H}_{3}$ The function $\mathrm{f}$ is continuous and there exists a positive constant $K$ such that

$D(f(t, x), f(t, y)) \leq K D(x, y)$ for all $x, y \in C\left(J, E^{l}\right)$.

If the assumptions $\mathrm{H}_{1}-\mathrm{H}_{3}$ hold and

$$
\frac{(\mathcal{S}+K)\left(T-t_{0}\right)^{q}}{\Gamma(q+1)}<\frac{1}{2}
$$

then problem (2) has a unique solution on J.
Proof. Let $\mathbf{P}: C\left(J, E^{1}\right) \longrightarrow C\left(J, E^{1}\right)$ be the operator defined as follows:

$$
\begin{aligned}
\mathbf{P} x(t) \ominus_{g H} x_{0}= & \frac{1}{\Gamma(q)} \int_{t_{0}}^{t}(t-s)^{q-1} A(s) x(s) d s \\
& +\frac{1}{\Gamma(q)} \int_{t_{0}}^{t}(t-s)^{q-1} f(s, x(s)) d s .
\end{aligned}
$$

Let $\rho$ be a positive real number such that $p \geq 2(D$ $\left.\left(x_{0}, 0_{E^{1}}\right)+\left(f_{0}\left(T-t_{0}\right)^{q}\right) /(\Gamma(q+1))\right)$, where $f_{0}=D_{s}\left(f, 0_{E^{1}}\right)$.

We can prove that the operator $\mathbf{P}$ transforms the ball $B_{\rho}\left(0_{E^{1}}, \rho\right)$ into itself, where

$$
B_{\rho}\left(0_{E^{1}}, \rho\right)=\left\{y \in C\left(\left[J, E^{1}\right): D\left(0_{E^{1}}, y\right) \leq \rho\right\} .\right.
$$

For this purpose, let $x \in B_{\rho}$; then, we have

$$
\begin{aligned}
& D\left(\mathbf{P} x(t), 0_{E^{1}}\right) \leq D\left(x_{0}, 0_{E^{1}}\right)+\frac{\mathcal{S}}{\Gamma(q)} \int_{t_{0}}^{t}(t-s)^{q-1} D\left(x(s), 0_{E^{1}}\right) d s \\
&+\frac{1}{\Gamma(q)} \int_{t_{0}}^{t}(t-s)^{q-1} D\left(f(s, x(s)), 0_{E^{1}}\right) d s, \\
& D\left(\mathbf{P} x(t), 0_{E^{1}}\right) \leq D\left(x_{0}, 0_{E^{1}}\right)+\frac{\rho \mathcal{S}\left(T-t_{0}\right)^{q}}{\Gamma(q+1)}+\frac{\left(\rho K+f_{0}\right)\left(T-t_{0}\right)^{q}}{\Gamma(q+1)}, \\
& D\left(\mathbf{P} x(t), 0_{E^{1}}\right) \leq \rho .
\end{aligned}
$$

It follows that $\mathbf{P} B_{\rho} \subset B_{\rho}$.

For $y, x \in C\left(J, E^{1}\right)$, we have

$$
\begin{aligned}
D(\mathbf{P} y(t), \mathbf{P} x(t)) \leq & \frac{1}{\Gamma(q)} \int_{t_{0}}^{t}(t-s)^{q-1} D(A(s) y(s), A(s) x(s)) d s \\
& +\frac{K}{\Gamma(q)} \int_{t_{0}}^{t}(t-s)^{q-1} D(y(s), x(s)) d s .
\end{aligned}
$$

It follows that

$$
D(\mathbf{P} y(t), \mathbf{P} x(t)) \leq \frac{(\mathcal{S}+K)\left(T-t_{0}\right)^{q}}{\Gamma(q+1)} D_{s}(y, x),
$$

which implies that

$$
D_{s}(\mathbf{P} y, \mathbf{P} x) \leq \frac{(\mathcal{S}+K)\left(T-t_{0}\right)^{q}}{\Gamma(q+1)} D_{s}(y, x) .
$$

Thus,

$$
D_{s}(\mathbf{P} y, \mathbf{P} x) \leq \frac{1}{2} D_{s}(y, x)
$$

Finally, $\mathbf{P}$ is a contraction mapping and therefore, there exists a unique fixed point $x \in B_{\rho}\left(0_{E^{1}}, \rho\right)$ such that $\mathbf{P} x(t)=$ $x(t)$ which is the solution of problem (2). 


\section{Illustrative Example}

In this section, we give an example to illustrate the practical usefulness of the results that we establish in the paper. By using Theorem 27, we can solve the following fuzzy semilinear fractional evolution equation:

$$
\left\{\begin{array}{l}
{ }^{c} D_{0^{+}}^{2 / 3} u(t)=\frac{1}{20} e^{-t} u(t)+\frac{e^{-t}}{9+e^{t}} u(t), \quad t \in[0,1] . \\
u(0)=u_{0} .
\end{array}\right.
$$

We define the operator $A$ by.

$$
A(t)=\frac{1}{20} e^{-t} I
$$

where $I$ is the identity mapping defined on $E^{1}$.

And the function $f$ is given by $f(t, u)=\left(e^{-t} /\left(9+e^{t}\right)\right) u(t)$

It is clear that $A(t)$ is a bounded linear operator and the function $t \longrightarrow A(t)$ is continuous. Hence, the assumptions $H_{1}$ and $H_{2}$ are verified.

On the other hand, we have

$$
\begin{aligned}
D(f(t, u(t)), f(t, v(t))) & =D\left(\frac{e^{-t}}{9+e^{t}} u(t), \frac{e^{-t}}{9+e^{t}} v(t)\right), \\
D(f(t, u(t)), f(t, v(t))) & \leq \frac{e^{-t}}{9+e^{t}} D(u(t), v(t)), \\
D(f(t, u), f(t, v)) & \leq \frac{1}{10} D(u, v) .
\end{aligned}
$$

Hence, the assumption $H_{3}$ in Theorem 27 holds with $K=1 / 10$

It remains to be checked that condition (32) in Theorem 27 is also satisfied with $t_{0}=0, T=1, K=1 / 10, \mathcal{S}=1 / 20$, and $\alpha=2 / 3$.

For this purpose, we have

$$
\begin{aligned}
\left(\frac{(K+\mathcal{S})\left(T-t_{0}\right)^{\alpha}}{\Gamma(\alpha+1)}\right) & =\left(\frac{((1 / 10)+(1 / 20)) \times(1-0)^{2 / 3}}{\Gamma((2 / 3)+1)}\right) \\
& =\frac{3}{20 \times 0.89}=0.16<\frac{1}{2}
\end{aligned}
$$

Finally, all the conditions of Theorem 27 are satisfied for problem (40); then, it has a unique solution on $[0,1]$.

Remark 28. We can show that the fuzzy semilinear fractional evolution equation (40) has a unique solution on $[0,1]$ for some fractional-order $0<\beta<1$. Indeed, we shall check that condition (32) in Theorem 27 is satisfied for some $\beta \in] 0,1[$. We have

$$
\left(\frac{(K+\mathcal{S})\left(T-t_{0}\right)^{\beta}}{\Gamma(\beta+1)}\right)<\frac{1}{2} \Longleftrightarrow \Gamma(\beta+1)>\frac{3}{10}
$$

Then, by using Theorem 27, the fuzzy semilinear fractional evolution equation (40) has a unique solution on $[0,1]$ for the values of $\beta$ satisfying (44).

\section{Conclusion and Future Work}

In this manuscript, we studied the existence and uniqueness results of fuzzy linear and semilinear fractional evolution equations involving Caputo fractional derivative. The existence theorems are proved by using fuzzy fractional calculus, Picard's iteration method, and Banach fixed-point theorem. As application, we present an illustrative example to show the applicability of our main results.

Our future work is to extend the obtained results in this paper to the infinite dimensional case by using the theory of fuzzy operator semigroups.

\section{Data Availability}

The data used to support the findings of this study are included in the references within the article.

\section{Conflicts of Interest}

The authors declare that they have no conflicts of interest.

\section{References}

[1] N. V. Hoa, H. Vu, and T. M. Duc, "Fuzzy fractional differential equations under Caputo-Katugampola fractional derivative approach," Fuzzy Sets and Systems, vol. 375, pp. 70-99, 2018.

[2] M. Mazandarani and A. V. Kamyad, "Modified fractional Euler method for solving fuzzy fractional initial value problem," Communications in Nonlinear Science and Numerical Simulation, vol. 18, no. 1, pp. 12-21, 2013.

[3] M. L. Puri and D. A. Ralescu, "Differentials of fuzzy functions," Journal of Mathematical Analysis and Applications, vol. 91, no. 2, pp. 552-558, 1983.

[4] R. P. Agarwal, M. Belmekki, and M. Benchohra, "A survey on semilinear differential equations and inclusions involving Riemann-Liouville fractional derivative," Advances in Difference Equations, vol. 2009, Article ID 981728, 47 pages, 2009.

[5] M. Caputo, "Linear models of dissipation whose Q is almost frequency independent," International Journal of Geographical Information Science, vol. 13, pp. 529-539, 1967.

[6] K. Diethelm, The Analysis of Fractional Differential Equations, Springer-Verlag, Berlin, 2010.

[7] F. Mainardi, Fractals and Fractional Calculus Continuum Mechanics, Springer Verlag, 1997.

[8] I. Podlubny, Fractional Differential Equations, Mathematics in Science and Engineering, New York, 1999.

[9] R. P. Agarwal, V. Lakshmikantham, and J. J. Nieto, "On the concept of solution for fractional differential equations with uncertainty," Nonlinear Analysis: Theory, Methods \& Applications, vol. 72, no. 6, pp. 2859-2862, 2010. 
[10] S. Arshad and V. Lupulescu, "On the fractional differential equations with uncertainty," Nonlinear Analysis, vol. 74, no. 11, pp. 3685-3693, 2011.

[11] R. Alikhani and F. Bahrami, "Global solutions for nonlinear fuzzy fractional integral and integrodifferential equations," Communications in Nonlinear Science and Numerical Simulation, vol. 18, no. 8, pp. 2007-2017, 2013.

[12] T. Allahviranloo, A. Armand, and Z. Gouyandeh, "Fuzzy fractional differential equations under generalized fuzzy Caputo derivative," Journal of Intelligent \& Fuzzy Systems, vol. 26, no. 3, pp. 1481-1490, 2014.

[13] S. Salahshour, T. Allahviranloo, S. Abbasbandy, and D. Baleanu, "Existence and uniqueness results for fractional differential equations with uncertainty," Advances in Difference Equations, vol. 112, 1847 pages, 2012.

[14] H. V. Ngo, V. Lupulescu, and D. O'Regan, "A note on initial value problems for fractional fuzzy differential equations," Fuzzy Sets and Systems, vol. 347, no. 347, pp. 54-69, 2018.

[15] S. Salahshour, T. Allahviranloo, and S. Abbasbandy, "Solving fuzzy fractional differential equations by fuzzy Laplace transforms," Communications in Nonlinear Science and Numerical Simulation, vol. 17, no. 3, pp. 1372-1381, 2012.

[16] X. B. shu and Y. Shi, "A study on the mild solution of impulsive fractional evolution equations," Applied Mathematics and Computation, vol. 273, no. 273, pp. 465-476, 2016.

[17] X. Ma, X. B. Shu, and J. Mao, "Existence of almost periodic solutions for fractional impulsive neutral stochastic differential equations with infinite delay," Stochastics and Dynamics, vol. 20, no. 1, article 2050003, 2020.

[18] Y. Guo, M. Chen, X. B. Shu, and F. Xu, "The existence and Hyers-Ulam stability of solution for almost periodical fractional stochastic differential equation with $\mathrm{fBm}$," Stochastic Analysis and Applications, vol. 39, no. 4, pp. 643-666, 2021.

[19] H. V. Long, N. T. K. Son, and H. T. T. Tam, "The solvability of fuzzy fractional partial differential equations under Caputo gH-differentiability," Fuzzy Sets and Systems, vol. 309, no. 309, pp. 35-63, 2017.

[20] H. V. Long, "On random fuzzy fractional partial integrodifferential equations under Caputo generalized Hukuhara differentiability," Computational and Applied Mathematics, vol. 37, no. 3, pp. 2738-2765, 2018.

[21] L. A. Zadeh, "Fuzzy sets," Information and Control, vol. 8, no. 3, pp. 338-353, 1965.

[22] B. Bede and L. Stefanini, "Generalized differentiability of fuzzy-valued functions," Fuzzy Sets and Systems, vol. 230, no. 230, pp. 119-141, 2013.

[23] H. T. Nguyen, "A note on the extension principle for fuzzy sets," Journal of Mathematical Analysis and Applications, vol. 64, no. 2, pp. 369-380, 1978.

[24] B. Bede, Mathematics of Fuzzy Sets and Fuzzy Logic, Springer, London, 2013.

[25] O. Kaleva, "Fuzzy differential equations," Fuzzy Sets and Systems, vol. 24, no. 3, pp. 301-317, 1987.

[26] D. Dubois and H. Prade, Fuzzy Sets and Systems: Theory and Applications, vol. 144, Academic Press, New York, 1980.

[27] A. Pazy, Semigroups of Linear Operators and Applications to Partial Differential Equations, Springer-Verlag, New York, 1983. 